\title{
COVERAGE STRATEGY FOR HETEROGENEOUS NODES IN WIRELESS SENSOR NETWORK BASED ON TEMPORAL VARIABILITY OF FARMLAND
}

\author{
Huarui $\mathrm{Wu}, \mathrm{Li} Z \mathrm{Zhu}$
}

Original scientific paper Given temporal variability of farmland, the coverage rate of wireless sensor network (WSN) is usually low. There may arise the problems of blind spot area and congestion of hot spots. We propose a coverage strategy for heterogeneous nodes in WSN based on temporal variability of farmland, which predicts the key nodes using key node prediction model according to temporal variability of farmland environment. Through introduction of renewable energy nodes, the positions of heterogeneous nodes in the network can be determined. The task is re-allocated to the heterogeneous nodes depending on the residual energy of nodes, and the state of nodes in the network is adjusted dynamically. Simulation shows that CSHN can effectively reduce the node death rate and network energy consumption, while prolonging the survival of network and equalizing coverage and network energy consumption.

Keywords: coverage rate; heterogeneous node; network energy consumption; temporal variability of farmland; Wireless Sensor Network (WSN)

Strategija pokrivenosti za heterogene čvorove u bežičnoj mreži senzora na temelju vremenske promjenljivosti poljoprivrednog zemljišta

Izvorni znanstveni članak

Ako se razmotre različiti vremenski uvjeti na poljoprivrednom zemljištu, stopa pokrivenosti bežične mreže senzora (WSN - Wireless Sensor Network) obično je mala. Mogu se pojaviti problemi slijepih točaka i prenatrpanosti vrućim točkama. Predlažemo strategiju pokrivenosti za heterogene čvorove u WSN na temelju vremenskih promjena koje utječu na obradivost, a koja predviđa ključne čvorove primjenom modela predviđanja ključnog čvora u skladu s vremenskim promjenama na imanju. Uvođenjem obnovljivih čvorova energije, mogu se odrediti položaji heterogenih čvorova u mreži. Zadatak se ponovo prebacuje na heterogene čvorove ovisno o zaostaloj energiji čvorova te se stanje čvorova u mreži dinamički podešava. Simulacija pokazuje da CSHN može učinkovito smanjiti stopu propadanja čvora i potrošnju energije mreže i tako produžiti opstojnost mreže te ujednačiti pokrivenost i potrošnju energije mreže.

Ključne riječi: bežična mreža senzora (WSN); heterogeni čvor; potrošnja energije mreže; stopa pokrivenosti; vremenska promjenljivost poljoprivrednog zemljišta

\section{Introduction}

Wireless sensor network (WSN) requires no establishment of infrastructures and integrates sensor technology, modern network technology and other hi-tech that renders high performance [1, 2]. WSN deploys sensors to acquire information and performs information fusion by embedded computing. For data transmission between the nodes, multi-hop technique and distributed information processing are usually used [3]. WSN has the advantages of low energy consumption, low cost, easy deployment, management and maintenance and flexible communications. WSN is composed of hundreds or thousands of nodes, with each node constituted by wireless communication module, processor module, sensor module and energy supply module. The nodes can be randomly or intentionally deployed, and the information of the object to be monitored in the covered area is collected and processed via wireless communications [4]. The operation of WSN needs no human work and can be done by self-organization mechanism. However, the electric energy available for running the WSN is limited, especially when there is no man to attend it or when WSN operates in adverse environment. It may be impossible to replace the power supply repeatedly, and to be able to work for a long time [5], WSN needs to have a low energy consumption and long service life.

In order to improve the performance and coverage of WSN, the strategy of dense deployment is usually adopted. But this has the problem of creating abundant nodes as well as abundant data collection, wireless channel interference, and high energy consumption due to aggravated channel conflict. Density control is to make some redundant nodes dormant, thus reducing network redundancy and network energy consumption and prolonging service life. Most of the current density control algorithms rely on external equipment for acquiring geographical information of the nodes and calculating the covering relations of the nodes. However, this method not only increases the network operating cost, but also has difficulty in acquiring accurate information of redundant nodes when the positioning information is less correct. As a consequence, there may be blind spot area and a reduction of coverage rate. More and more researches on coverage control [6, 7] have been done recently, there are two main methods: One is research node scheduling strategy, which puts redundancy nodes take turns into dormant state; The other is research network coverage hole detection and repair strategy, which imagines the sensor node perception range as a circle based on Voronoi and divided monitoring area into multiple Voronoi polygon according to sensor node location information. So, coverage control problem has changed into the famous geometry problem. Literature [8] carried out calculation of covering relations of nodes without precise knowledge of geographical positions of nodes. The results of network detection are inaccurate because of calculation errors and presence of blind spot area. Therefore, node deployment strategy of WSN without knowing the geographical information of nodes is the topic under intensive study at present. The solutions to this problem are to precisely locate the redundant nodes and obtain the minimum cover set so as to achieve 
optimal node deployment. C. Liu et al. [9] randomly divided sensor nodes into $\mathrm{k}$ subsets with each node periodically performing the sensing task. But their method has the problem of unpredictable coverage rate due to randomness of subset division. Literature [10] was concerned with node deployment and coverage in circular area. The minimum number of nodes was determined for the entire monitored area by calculating the inclusion angle between the circle and the monitored area. Though the number of redundant nodes was decreased and the coverage rate was high, the network connectivity was reduced. WSN is usually seen in adverse environment, where battery replacement is difficult. Given the limited electric power supplied by conventional batteries, other forms of energy can be utilized, including solar energy, wind energy, heat energy, mechanical vibration energy, acoustic energy and electromagnetic energy. Some studies have been conducted on how to harvest these forms of energy, and progress is made in harvesting mechanical vibration energy and light energy in particular. The idea of utilizing renewable energy has been proposed for WSN, and WSN in agriculture utilizing solar energy is among the research hotspots. The coverage rate is an important parameter of WSN obtained integrity and nonrepudiation information. This reflects WSN perception ability, which was used to describe the quality of service [11-13]. In order to achieve full coverage of the monitor area, the most usual way to improve the coverage rate of wireless sensor network is dense deployment. In this deployment strategy, coverage rate was assured, but there is perception coverage intersection between nodes in the network, which will cause some problems such as information data redundancy acquisition, channel conflict and so on. Meanwhile, input-output ratio was considered in the environmental monitoring, so the dense deployment strategy is not usually adopted when the fund is insufficient. In the development of agriculture, WSN has low power consumption and low cost, which has been widely applied to real-time monitoring for large-scale farmland production. The monitoring effectiveness depends on the coverage ratio of the wireless sensor network, the continuous working time, the data transmission time and some other factors. Therefore, the coverage strategy of wireless sensor nodes and other factors is very important to the monitoring quality. The coverage characteristics of wireless sensor node are affected by temporal variability of farmland such as the crop planting density and height, the foliage density, etc. We propose a coverage strategy for heterogeneous nodes in WSN based on temporal variability of farmland with the goal of improving the coverage rate. First we establish the key node prediction model with the factors of path loss $\mathrm{W}$, sensing probability of node $P$, effective sensing area $S$ and residual energy $Q$. Thus, the attribute $x$ of key nodes of WSN is determined. The network maintains good connectivity after topology changes of WSN. The nodes utilizing renewable energy are introduced. These nodes can achieve energy self-supply by harvesting solar energy as well as automatic management of recharging and energy storage. Therefore, the energy saving performance is adjusted dynamically. Using this prediction model, the performance of the key nodes is analysed. Depending on the temporal and spatial variability of farmland environment, total number of nodes, proportions of ordinary nodes and nodes utilizing renewable energy, positions of heterogeneous nodes and node importance are converted into linear constraint conditions. The blind spot area and congestion of hot spots can be avoided by reasonably deploying each type of nodes and optimizing the positions of heterogeneous nodes. In the meantime, the coverage rate of WSN is increased. In order to equalize the network energy consumption and considering the difference in energy supply between ordinary nodes and nodes utilizing renewable energy, the task is re-allocated to the heterogeneous nodes based on residual energy of nodes combining the mechanism of interactive deployment of heterogeneous nodes. Through dynamic adjustment, the network energy consumption is equalized with lower node death rate, lower unnecessary energy consumption and longer service life.

\section{Network model and problem description 2.1 Network model}

$N$ nodes of WSN are randomly and evenly deployed in a $2 \mathrm{D}$ monitored area $A$, which is assumed to possess the following features:

(1) The nodes are heterogeneous in that ordinary nodes and nodes utilizing solar energy are deployed. These nodes constitute the wireless self-organizing network.

(2) The node communication radius $R_{\mathrm{c}}$ has the following relation with the maximum sensing radius $R_{\mathrm{s}}$ : $R_{\mathrm{c}} \geq 2 R_{\mathrm{s}}$.

(3) The initial energy of all nodes is $W$ and there are synchronized clocks.

(4) The boundary effect of the monitored area is negligible with high node density.

(5) The precise positions of the nodes in the network are known, and the nodes can acquire the information of the neighbouring nodes within the effective communication radius, including residual electric power and positions.

\subsection{Problem description}

A large amount of nodes are randomly and evenly deployed within monitored area $A$. The node set is $S$, and the minimum coverage rate is $\eta$. Based on differentiation of the monitored area, the key node set $a$ is found. Thus the heterogeneous nodes are reasonably deployed to prolong the service life and minimize energy consumption.

Definition 1 (redundant nodes)

Within monitored area $A$, there are nodes $S_{i}$ and $S_{j}$, $i \neq j$. If the sensing area of $S_{i}$ and $S_{j}$ has the following relation, i.e. $\Omega_{1} \cap \Omega_{2}=\Omega_{1}$, where $\Omega_{1}$ is the effective sensing area of $S_{j}$ in area $A$ and $\Omega_{2}$ is the effective sensing area of $S_{i}$, then $S_{j}$ is considered redundant.

Definition 2 (node redundancy)

Consider any node $S_{i}$ and the set $S_{0}$ of its neighbour nodes within the monitored area. Let the sensing area of $S_{i}$ be $\varphi$, the set of sensing area of S0 be $\tau=\left\{\tau_{1} \ldots \tau_{n}\right\}$, and node redundancy of $S_{i}$ be $\Gamma$. 
$\Gamma=\frac{\left(\varphi \bigcap \tau_{1}\right) \bigcup\left(\varphi \cap \tau_{2}\right) \ldots . .\left(\varphi \cap \tau_{n}\right)}{\varphi}=\frac{\sum_{i=1}^{n}\left(\varphi \cap \tau_{i}\right)}{\varphi}$

Definition 3 (coverage rate)

Coverage rate $\eta$ is the ratio of effective coverage area of all nodes within monitored area $\mathrm{A}$ to total monitored area A. It is an important indicator of network coverage performance.

$\eta=\frac{\sum_{i=1}^{n} \xi(i)}{A}$

Definition 4 (set of sensing neighbours)

For any node $S_{i}$, the set of its sensing neighbours is defined as

$\psi(i)=\left\{S_{j} \in S \mid d\left(S_{i}, S_{j}\right) \leq R_{i}+R_{j}, S_{i} \in S, j \neq i\right\}$

where $S$ is the set of nodes deployed within area $A$; $d\left(S_{i}, S_{j}\right)$ is the Euclidean distance between $S_{i}$ and $S_{j} ; R_{i}$ and $R_{j}$ are the sensing radius between $S_{i}$ and $S_{j}$.

Definition 5 (coverage balance degree)

An appropriate coverage balance degree means less energy consumption and prevents earlier failure of nodes due to unequalised task and energy allocation.

$$
\Gamma=\frac{\sum_{i=1}^{n} \sqrt{\frac{1}{m_{i}} \sum_{j=1}^{m_{i}}\left[d(i, j)-\hbar_{i}\right]^{2}}}{N}
$$

where $\Gamma$ is coverage balance degree; $N$ is total number of nodes; $d(i, j)$ is the Euclidean distance between $S_{i}$ and $S_{j}$; the number of neighbour nodes of $S_{i}$ is $\mathrm{mi} ; \hbar_{i}$ is the average distance between $S_{i}$ and $S_{k}$; the sensing area of $S_{i}$ and $S_{k}$ overlaps.

Definition 6 (blind spot area)

For monitored area $A$, if there is node set $\Theta \subseteq A$, but $\Theta$ is not sensed by a node in $S$, then $\Theta$ is defined the blind spot area.

\subsection{Key node prediction model}

Network connectivity is an important performance indicator of WSN. As WSN operates, key node failure, abrupt increase of data traffic and natural disasters will occur $[14 \div 16]$. As a result, the network link will break and the communication efficiency decreases. These emergencies are closely related to the monitored environment. The farmland has certain temporal and spatial variability, and how to locate the key nodes to cope with emergencies is crucial for improving network coverage. The key nodes of WSN are the nodes that influence network connectivity and whose effectiveness determines network segmentation. The nodes start with the same initial state, and as time progresses, there will be differences in the monitored environment and allocated task, leading to differences in state of nodes. Based on spatial and temporal variability of farmland environment, we classify the monitored area and determine the key nodes that have a constraint on network performance. Thus the set of key nodes and the key node prediction model are established. In ideal situations, the propagation loss increases with the transmission distance:

$l=\frac{P_{\mathrm{s}}}{P_{\mathrm{r}}}=\left(\frac{4 \tau f d}{c^{2}}\right)^{2}$

where $l$ is propagation loss; $P_{\mathrm{s}}$ is transmission power; $P_{\mathrm{r}}$ is received power; $d$ is the distance between the transmitting node and the receiving node. Considering temporal and spatial variability of farmland, the path loss of signals during propagation is expressed as

$w=10 n \lg \left(\frac{4 \pi}{\lambda}\right)+10 n \lg d+f\left(h, d_{h}, v\right)$

where $w$ is intensity of path loss; $f\left(h, d_{h}, v\right)$ is environmental factor function related to temporal variability; $h$ is the height of crops; $d$ is the distance between crops; $v$ is crop density.

For monitored area $A$ where node $S_{i}$ is located, the set of its neighbour nodes is $G$; For any point a in area $A$, the probability of being sensed by a node in $G$ is expressed as

$P=\frac{R_{0}^{2}}{4\left(R_{0}^{2}-\sigma^{2}\right)}$

Effective coverage of WSN is related to the sensing probability for the area being monitored. As the signals propagate, the signal intensity will decrease with the increasing propagation distance. For extensive crop growing area, the changing growth status of the crops will lead to changes of the entire environment being monitored. The indicators of crops, including density, height and leaf area, will all influence wireless signal transmission and network coverage. Consider a point a within monitored area A. If this point can be sensed by a node $S$, then this node is considered effective and the wireless signals of this node can be received. Let $w_{k}$ be the path loss threshold. If the path loss of relevant node is less than $w_{k}$, this means the point cannot be sensed by the nodes and the signals are not effectively received. Thus this node is ineffective, and the point represents the blind spot area.

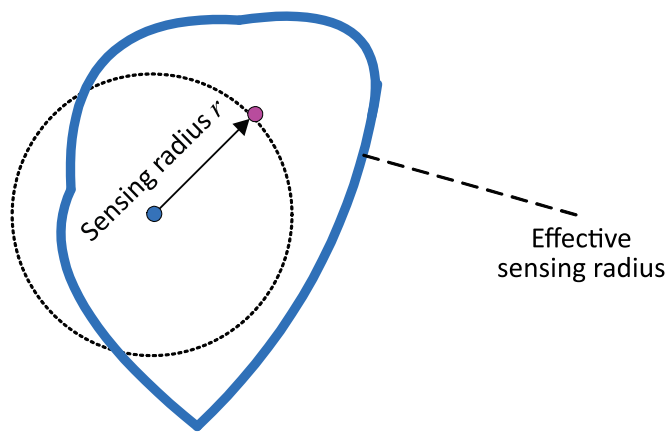

Figure 1 Effective sensing radius of node 
The effective sensing area of point a is estimated as follows. The effective sensing probability of node $b$ for point $\mathrm{a}$ is $p(a)$, and several nodes similar as $\mathrm{b}$ constitute the effective sensing area of point a. However, this effective sensing area is not a regular circle that is otherwise supposed in ideal situation. Thus the effective sensing area $S$ is expressed as

$$
\begin{aligned}
S & =\int_{0}^{2 \pi} \int_{0}^{\infty} p(r) r \mathrm{~d} r \mathrm{~d} \varphi= \\
& =2 \pi \int_{0}^{\infty}\left(1-f\left(h, d_{h}, v\right)+\frac{\Phi}{\sqrt{2}} \lg \left(\frac{r}{r_{0}}\right)\right) r \mathrm{~d} r \mathrm{~d} \varphi
\end{aligned}
$$

where $r_{0}$ is sensing radius of node; $\Phi$ is attenuation factor due to temporal variability of environment. The larger the $\Phi$, the greater the influence of environmental changes on signal propagation will be. The main factors influencing network coverage are path loss $W$, sensing probability $P$, effective sensing area $\mathrm{S}$ and residual energy $Q$ of the node. These factors constitute the attribute $x$ of key nodes.

$\chi=\alpha W+\beta S+\gamma Q+\delta P$

where $\alpha, \beta, \gamma$ and $\delta$ are the weights of attribute factors, and $\alpha+\beta+\gamma=1$. When the spacing between the crops is large, the weight of sensing probability is higher; when the crop growth is faster and the leaf area index is larger, the path loss has a higher weight. The attributes of key nodes in WSN are in direct proportion to network coverage. When the nodes are sparse, the network cost can be decreased by adjusting weight and the number of nodes; when the nodes are dense, the interactive deployment of nodes can be invoked to reduce cost and the path loss arising from channel interference.

\section{Coverage strategy}

According to the key node prediction model, the growth status of crops has considerable impact on wireless signal transmission, which also shows spatial and temporal variability between nodes as they operate. This will lead to changes of network topography and network connectivity. For WSN, node energy is the decisive factor that relates to all these problems, and nodes utilizing renewable energy are introduced here. However, this creates heterogeneity of nodes. Combining key node prediction model, energy supply features of two nodes and network connectivity, we propose a deployment strategy for the heterogeneous nodes. This strategy aims to reduce total number of nodes, coverage redundancy and blind spot area and to improve network performance.

Letting $N(t)$ be the total number of nodes at time $t$,

$$
N(t)=\int_{0}^{t} r \Gamma(\varpi, \xi) \mathrm{d} t
$$

where $\varpi$ and $\xi$ are the proportions of ordinary nodes and nodes utilizing renewable energy, respectively, and $\varpi+\xi$ $=1$.

$$
\Gamma(\varpi, \xi)=\lim _{t \rightarrow \infty} \frac{1}{E[N(t)]} \sum_{i=1, j=1}^{\infty} r f\left(h, d_{h}, v\right) \varpi_{i} \xi_{j}
$$

It can be known that $\varpi$ and $\xi$ vary within the interval $(0$, $1)$. When $\varpi \rightarrow 0$ and $\xi \rightarrow 1$, the network has good correction capacity on redundancy; when $\varpi \rightarrow 1$ and $\xi \rightarrow$ 0 , the network has good connectivity. Thus, redundancy and blind spot area can be balanced by adjusting $\varpi$ and $\xi$ to optimize connectivity and area coverage. To ensure network connectivity, the minimum path length $L$ between nodes needs to be related to the average distance $d_{i j}$ between ordinary node and node utilizing renewable energy by the following formula:

$$
L=\frac{\eta}{N(N-1)} \sum_{i \neq j} d_{i j}
$$

The role played by heterogeneous nodes has to change constantly. Letting node importance be $\mathfrak{R}_{\mathrm{i}}$ and node degree of node $i$ be $\hbar_{i}$,

$$
\mathfrak{R}_{i}=\chi_{i} \frac{\hbar_{i}}{\sum_{i=1}^{n} \hbar_{i}} \Theta(\varpi, \xi, t)
$$

Depending on spatial and temporal variability of farmland, total number of nodes, proportion of ordinary nodes and nodes utilizing renewable energy, positions of heterogeneous nodes and node importance are converted into linear constraints. By constraint tightening, the feasible solution obtained is the deployment of heterogeneous nodes.

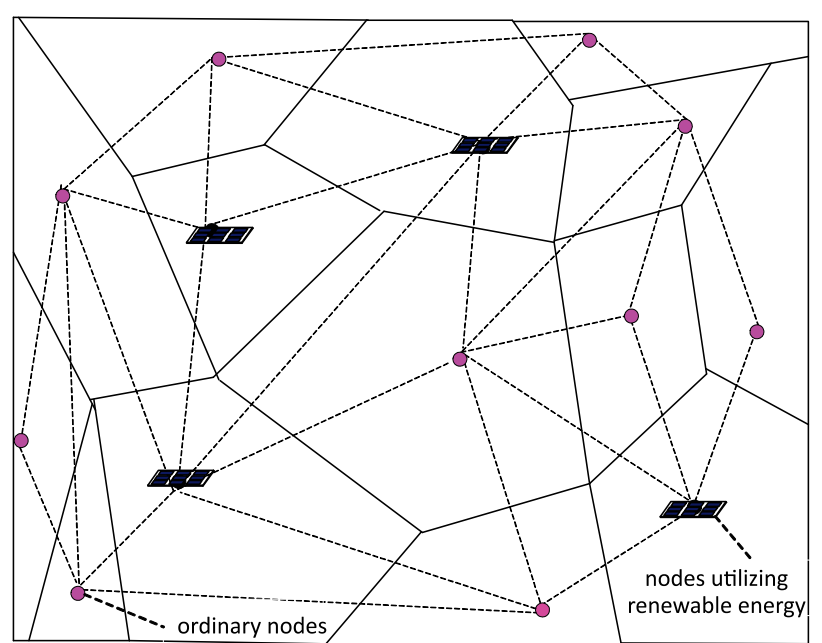

Figure 2 Deployment of heterogeneous nodes in WSN

To equalize network energy consumption with heterogeneous nodes, the interactive deployment of nodes is conducted. Two problems are considered: firstly, how the nodes determine the attributes of itself, i.e., redundant or not, and the residual energy; secondly, what is the deployment strategy for the redundant node that can prolong the service life and reduce energy consumption of the network on the basis of ensuring coverage. 
The attributes of redundant nodes are analysed, including both ordinary nodes and nodes utilizing renewable energy. The redundancy of nodes is first determined according to the relation between residual energy of nodes and network coverage, and then the redundant node set is obtained. Thus the state of the redundant nodes is adjusted based on residual energy. Some redundant nodes are made dormant or shifting between different state according to network operation and node redundancy. The deployment of redundant nodes is performed as follows according to residual energy and current coverage:

Step 1 Determine the redundant node set and acquire the residual energy of nodes.

Step 2 On Delaunay triangulation pattern G(Si, E, V) for node set, the non-redundant nodes and the sides connecting to these nodes are removed using redundant node set. Thus a new pattern is obtained. If the redundant nodes are made dormant, the adjacent redundant nodes are dormant as well. As a result, there will be blind spot area in WSN. The selection of redundant nodes should be based on the area coverage of nodes.

Step 3 The expectation of redundancy is related to the number of neighbour nodes of the current node to be made dormant. Thus the nodes having a larger number of redundant neighbour nodes are preferred. If the two nodes have equal number of redundant neighbour nodes, the node $v_{k}$ that has a lower residual energy is made dormant, and this node is placed (replicated) into the set of dormant nodes Q; otherwise, the node that has a higher residual energy is made dormant. However, this may lead to earlier death of other nodes due to excess task allocated to them, resulting in lower network connectivity and shorter service life.

Step 4 Repeat the above steps until all nodes are transversed. The nodes included in set $\mathrm{Q}$ are nodes that can be made dormant; nodes in $\mathrm{V}-\mathrm{Q}$ constitute the connected cover set based on equalized energy consumption for area $\mathrm{T}$.

\section{Simulation experiment}

We perform simulation experiment to compare the performance of different algorithms, the simulation tool use MATLAB. The experiment scenarios are configured as follows: there are 120 nodes in the area of $100 \times 100$ $\mathrm{m}^{2} ; 100$ are ordinary nodes and the remaining 20 are nodes utilizing solar energy. The nodes have consistent initial energy $2 \mathrm{~J}$; the maximum communication radius of nodes $R_{\mathrm{c}}=20 \mathrm{~m}$, while the sensing radius is variable. The operating state of the nodes is sampled at different time points, and the performance of the algorithm is measured by 1 ) network connectivity; 2) total number of nodes; 3 ) residual energy of nodes; 4) quality of coverage.The strategy this paper proposed is named after CSHN (coverage strategy for heterogeneous nodes in WSN based on temporal variability of farmland) for convenient. CSHN is compared with the distributed random algorithm.

Connectivity is the core aspect of WSN and the precondition for communications of all nodes. After collaboration between nodes for some time, connectivity will change due to differentiation of monitored environment and operating state. It can be seen from Fig. 3(b) that CSHN algorithm has a better connectivity with uniform distribution of nodes. Because of differences in deployment strategy, the difference in connectivity is expanding, and the gain in performance of nodes is gradually increasing. The increase in connectivity is largely related to deployment under the same environmental parameters.

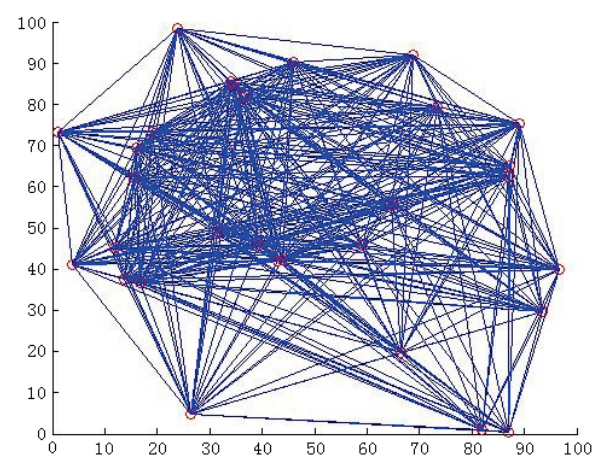

(a)

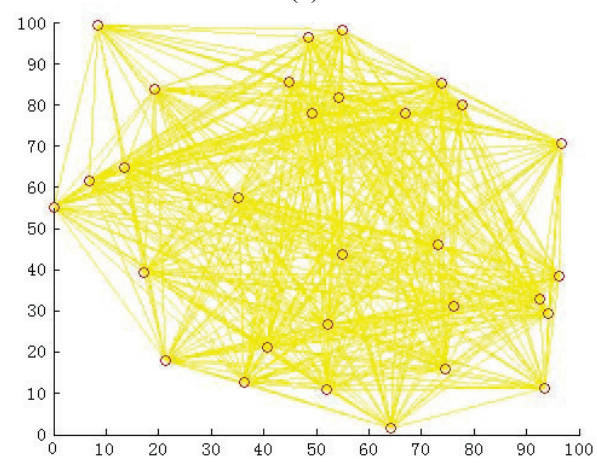

(b)

Figure 3 Network connectivity

Fig. 4 shows the variation of effective nodes in the network over time. The number of effective nodes is one key factor controlling the service life and performance of network. At the beginning of operation, the nodes have small difference, and the number of effective nodes changes little. But as time progresses, some nodes die because of tasks allocated to them and the variability of environment. This affects network stability. With the same coverage, CSHN algorithm and random algorithm begin to show different number of effective nodes. CSHN algorithm has a larger number of effective nodes and better extensibility.

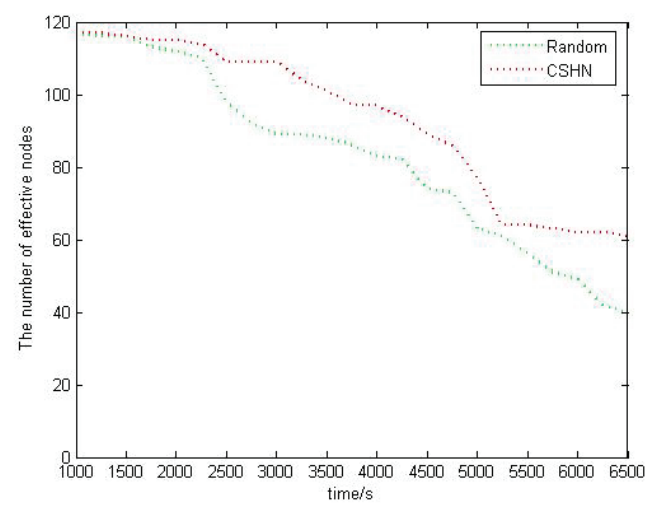

Figure 4 Effective nodes 
Network energy consumption rate is the ratio of energy lost to total energy and is one measure of network performance. Fig. 5 provides the variation of energy consumption rate for each algorithm. The two algorithms have similar energy consumption at the start, but as time progresses, CSHN algorithm shows superiority in energy consumption. With the introduction of nodes utilizing solar energy and prediction of key nodes depending on environment, the solar energy utilization efficiency is increased, and the distribution of energy consumption across the nodes is made more uniform. Early death of nodes is avoided, and the total energy consumption of network is decreased.

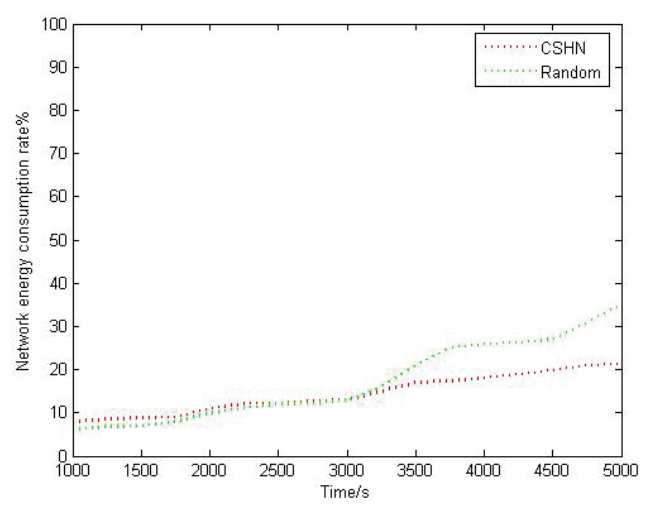

Figure 5 Residual energy

Coverage rate is an important indicator of coverage quality. Fig. 6 shows the relationship between coverage rate and service life of network. At the beginning of operation, the nodes are densely deployed with similar attributes and coverage rate. But due to a large number of redundant nodes, the energy is consumed at a fast rate. Some nodes die and the coverage rate decreases. CSHN algorithm achieves a dynamic adjustment of redundant nodes over time and establishes connected cover set based on equalized energy consumption; therefore, the survival nodes are more uniformly distributed. Compared with random algorithm, CSHN algorithm has a mild variation of coverage rate at later stage. The network performance is more stable over time, which satisfies the requirement in practical environment.

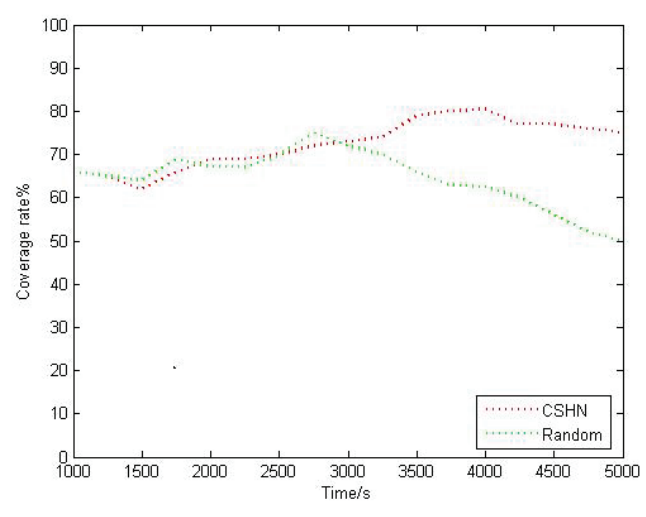

Figure 6 Quality of coverage

\section{Conclusion}

We propose a coverage strategy for heterogeneous nodes in WSN based on temporal variability of farmland.
A key node prediction mode is built and used to predict the sensitive area. The energy supply features of ordinary nodes and nodes utilizing renewable energy are considered during deployment with the focus laid upon reducing blind spot area and congestion of hot spots and increasing effective coverage. The interactive deployment of heterogeneous nodes is invoked to achieve collaboration between ordinary nodes and nodes utilizing renewable energy, equalization of energy consumption and reduction of energy consumption. Simulation experiment shows that with the same initial number of nodes, our method can more effectively reduce redundant nodes as compared with random; the nodes with less residual energy are allocated with less tasks to prevent failure, so as to improve connectivity, prolong the service life and equalize distribution of energy consumption. Using our algorithm, a reasonable balance is realized between coverage and energy consumption, with reduction of unnecessary energy consumption and increase of service life.

\section{Acknowledgements}

The work presented in this paper was supported by the National Natural Science Foundation of China (Grants No. NSFC-61271257) and the National Natural Science Foundation of China (Grants No.NSFC-61471067).

\section{References}

[1] Nguyen, D. T.; Nguyen, N. P.; Thai, M. T.; Helal A. Optimal and distributed algorithms for coverage hole healing in hybrid sensor networks. // International Journal of Sensor Networks. 4, 11(2012), pp. 228-240. DOI: 10.1504/IJSNET.2012.047151

[2] Lambrou, T. P. Optimized Cooperative DynamicCoverage in Mixed Sensor Networks. // ACM Transactions on Sensor Networks. 3, 11(2015), pp. 312-319. DOI: 10.1145/2700260

[3] Ashouri, M.; Zali, Z.; Mousavi, S. R.; Hashemi, M. R. New optimalsolutions to disjoint set K-coverage for lifetimeextension in wireless sensor networks. // IET Wireless Sensor Systems. 1, 2(2012), pp. 31-39. DOI: 10.1049/iet-wss.2011.0085

[4] Tabirca, T.; Yang, L. T.; Tabirca, S. Smallest Number of Sensors for k-Covering. // International Journal of Computers Communications \& Control. 2, 8(2013), pp. 312-319. DOI: 10.15837/ijccc.2013.2.311

[5] Engels, A.; Reyer, M.; Xu, X.; Mathar, R. Autonomous self-optimization of coverage and capacity in LTE cellular networks. // IEEE Transactions on Vehicular Technology. 5, 62(2013), pp. 1989-2004. DOI: 10.1109/TVT.2013.2256441

[6] Mini, S.; Udgata, S. K.; Sabat, S. L. Sensor deployment and scheduling for target coverage problem in wireless sensor networks. // IEEE Sensors Journal. 3, 14(2014), pp. 636644. DOI: $10.1193 / 1.3159004$

[7] Abdulhalim, M. F.; Attea, B. A.Multilayergeneticalgorithm for maximum disjointreliable set coversproblem in wireless sensor networks. // Wireless Personal Communications. 1, 80(2015), pp. 203-227. DOI: 10.1007/s11277-014-2004-8

[8] Ye, F.; Zhong, G.; Lu, S.; Zhang, L. PEAS: A robust energy conserving protocol for long-lived sensor networks. // IEEE International Conference on Network Protocols. (2002), pp. 28-37. DOI: 10.1109/ICNP.2002.1181406

[9] Liu, C.; Wu, K.; Xiao, Y.; Sun, B. Random coverage with guaranteed connectivity: joint scheduling for wireless sensor networks. // IEEE Transactions on Parallel\& 
Distributed Systems. 6, 17(2006), pp. 562-575. DOI: 10.1109/TPDS.2006.77

[10] Khedr, A. M.; Osamy, W. Minimum perimeter coverage of query regions in a heterogeneous wireless sensor network. // Information Sciences. 15, 181(2011), pp. 3130-3142. DOI: 10.1016/j.ins.2011.04.008

[11] Vejarano, G.; Wang, D.; Dubey, R.; Mcnair, J. Distributed Throughput Maximization in Wireless Networks Using the Stability Region. // Parallel \& Distributed Systems IEEE Transactions on. 7, 25(2014), pp. 1713-1723. DOI: 10.1109/TPDS.2013.202

[12] Chanak, P.; Banerjee, I.; Rahaman, H. Load management scheme for energy holes reduction in wireless sensor networks. // Computers \& Electrical Engineering. 10, 48 (2015), pp. 343-357. DOI: 10.1016/j.compeleceng.2015.05.013

[13] Berger, A.; Pichler, M.; Haselmayr, W.; Springer A. Energy-efficient and reliable wireless sensor networks-An extension to IEEE 802.15. 4e. // EURASIP Journal on Wireless Communications and Networking. 1, 14(2014), pp. 1-12. DOI: 10.1186/1687-1499-2014-126

[14] Dai, L.; Chang, Y.; Shen, Z. An Optimal Task Scheduling Algorithm in Wireless Sensor Networks. // International Journal of Computers Communications \& Control. 1, 6(2011), pp. 312-319. DOI: 10.15837/ijccc.2011.1.2205

[15] Wang, D.; Xie, B.; Agrawal, D. P. Coverage and lifetime optimization of wireless sensor networks with Gaussian distribution. // IEEE Trans. on Mobile Computing. 12, 7(2008), pp. 1444-1458. DOI: 10.1109/TMC.2008.60.

[16] Hossain, A.; Chakrabarti, S.; Biswas, P. K. Impact of sensing model on wireless sensor network coverage. // IET Wireless Sensor Systems. 3, 2(2012), pp. 272-281. DOI: 10.1049/iet-wss.2011.0101

\section{Authors' addresses}

\section{Huarui $W u$}

Beijing Research Center for Information Technology in

Agriculture, Beijing Academy of Agriculture and Forestry Sciences Beijing 100097, China

National Engineering Research Center for Information Technology in Agriculture, Beijing 100097, China

Key Laboratories for Information Technology in Agriculture,

Beijing 100097, China

E-mail: wuhr@nercita.org.cn

Li Zhu, corresponding author

Beijing Research Center for Information Technology in

Agriculture, Beijing Academy of Agriculture and Forestry Sciences Beijing 100097, China

National Engineering Research Center for Information Technology in Agriculture, Beijing 100097, China

Key Laboratories for Information Technology in Agriculture,

Beijing 100097, China

E-mail: zhul@nercita.org.cn 